\title{
Conformational properties of block-polyampholytes adsorbed on charged cylindrical surfaces
}

\author{
Daniel L.Z. Caetano and Sidney J. de Carvalho ${ }^{\mathrm{a}}$ \\ Departamento de Física, Instituto de Biociências, Letras e Ciências Exatas, Universidade Estadual Paulista "Júlio de Mesquita \\ Filho" (UNESP), Rua Cristovão Colombo 2265, Jd. Nazareth, 15054-000, São José do Rio Preto, São Paulo, Brazil
}

Received 13 January 2017 and Received in final form 4 March 2017

Published online: 27 March 2017 - C EDP Sciences / Società Italiana di Fisica / Springer-Verlag 2017

\begin{abstract}
Polyampholytes are polymers that have positive and negative monomers along their chain. The adsorption of polyampholytes on charged surfaces has been the subject of a large number of theoretical, computational and experimental studies due to its importance in a variety of bio and nanothechnological systems. However, computational studies focusing on interaction between polyampholytes and cylindrical charged surfaces are rather scarce. This study, therefore, aims to investigate the conformational properties of block-polyampholytes in the presence of a negatively charged cylinder by means of Metropolis Monte Carlo simulations. Adopting a simplified model in which the electrolyte solution is treated at the DebyeHückel level, the effects of the ionic strength, the linear charge density of the cylinder and the block length on monomers distributions have been investigated. It was found that increasing the salt concentration promotes a transition from a conformation characterized by large loops to a necklace-like conformation parallel to the surface. It was also shown that, at low cylinder charge density, the increase in salt concentration and the length of the blocks lead to a change in the orientation of the adsorbed chain.
\end{abstract}

\section{Introduction}

The interaction between polyelectrolytes and charged surfaces is a subject which has attracted great attention in the last decades [1-6]. It is a central problem in statistical physics of macromolecules as well as a good model to study the interaction of polyelectrolytes with macromolecules like proteins and micelles [7-11]. Furthermore, such interest is also due to its wide applicability. One can highlight, for instance, the layer-by-layer deposition for encapsulation of drugs or biomolecules for purposes of drug delivery [12-18] and non-viral gene therapy [1922]. Particularly interesting are polyampholytes, which are polyelectrolytes that present both negative and positive charges [23]. In this kind of macromolecule, the charges can be distributed randomly along its chain or they can be grouped in blocks whose size is determined to yield desired properties [24]. The interaction between charged surfaces and block-polyampholytes can lead to conformations in which the blocks oppositely charged to the surface are adsorbed, and the other ones are stretched from the surface, which can promote colloidal stabilization [25-27]. In the case of DNA-polyampholytes interaction, this charged layer formed by negative monomers can avoid undesired interactions of DNA with proteins [28].

There are a number of theoretical studies addressing the conditions in which the polyelectrolytes-surface inter-

\footnotetext{
a e-mail: sidneyjc@ibilce.unesp.br
}

action is strong enough to get adsorption as well as the conformational states of the adsorbed chain [10,29-42]. In particular, the adsorption of a single random polyampholyte chain on a flat charged surface was addressed theoretically by Dobrynin and co-authors [43]. In this work, it was shown that adsorption can occur even when the polymer has a net charge with the same sign as that of the surface, in agreement with experiments [44]. Three adsorption regimes were identified with the increase of the surface charge density, $\sigma$. In the absence of salt and low $\sigma$, the polymer adopts an elongated conformation in the perpendicular direction to the surface, with size smaller than the Gouy-Chapman length $\lambda$ (pole regime). The increase of $\sigma$ leads to the formation of multiples loops with size about $\lambda$ (fence regime). In the pancake regime, $\lambda$ is smaller than the distance between two charged monomers and such a distance is responsible for the thickness of the adsorbed layer. With the addition of salt, the range of the surface electrostatic potential is defined by the Debye length, $\kappa^{-1}$, which goes on to determine not only the boundary for the pole regime, but also the thickness of the adsorbed layer in the fence regime when $\kappa^{-1}<\lambda$. For high enough salt concentration, the chain is bound to the surface in a weak adsorption regime in which the polymer does not undergo deformation.

Netz and Joanny have studied the interaction of random polyampholyte chains with planar, cylindrical and spherical charged surfaces [45]. They have gotten the same three regimes observed for plane geometry. The 
characteristic length that defines the boundary between these regimes, at low ionic strength, is not given by $\lambda$, but by the radius $R$ and $R / \xi$ for adsorption on spherical and cylindrical surfaces, respectively, where $\xi$ is the charge parameter defined by the Manning counterion condensation theory [46]. The effects of repulsive non-electrostatic monomer-monomer interaction were addressed by Zhulina, Dobrynin and Rubinstein [47-50]. For spheres smaller than the unperturbed polymer size, they have found that the dominance of these short-range interactions leads to a pseudo-brush conformation with thickness smaller than $R$. With the further decrease of the radius, the chain adopts a pseudo-star conformation in which the thickness is larger than the radius [47].

Computational simulation studies have been presented with several purposes. Khan and co-authors have investigated the adsorption of flexible polyampholytes between two charged planar surfaces by means of Metropolis Monte Carlo simulations [51]. The general qualitative behavior obtained from analytical theories was reproduced, but the pancake regime was not recovered. The specific case of block-polyampholytes was addressed by Messina, where he has assessed the effects of block length and surface charge density on the monomer distribution [52]. The adsorption of polyampholytes on a spherical nanoparticle was addressed by Feng and Ruckenstein, who showed that the conformation of the adsorbed polyampholyte chain is dependent on the charge density and particle size [53]. They have found, as in this study, that the strong repulsion between the particle and like-charged monomers leads to an increase of thickness of the adsorbed layer with the increase in $\sigma$, in disagreement with theory [43]. Also considering spherical geometry, Stoll and co-authors have studied the influence of various parameters, such as $p \mathrm{H}$, ionic strength, number and size of the blocks and surface charge density on adsorption of weak polyampholyte chains [54, 55]. Using Brownian dynamics, Chen and Ruckenstein have investigated changes in the structure of complexes composed of a hydrophobic nanoparticle and a multiblock copolymer molecule [56].

Despite these efforts, results from computational simulations focusing on the interaction between cylindrical surfaces and block-polyampholytes were not reported. In order to fill this gap and extend previous predictions for random copolymers, the purpose of the present study is to investigate the adsorption of block-polyampholytes onto a charged cylinder by means of Metropolis Monte Carlo simulations. The effects of salt concentration, cylinder charge density and block length were investigated for the adsorbed polymer. We observe that the increase of salt concentration leads from a conformation characterized by large loops to a necklace-like conformation. This last conformation is parallel to the surface for highly charged cylinders and becomes normal to the surface for decreasing charge density.

\section{Model and simulations}

We model the system by one infinite rigid cylinder and one polyampholyte chain, both confined within an electrically neutral cylindrical cell $[57,58]$. The inner cylinder was kept fixed at the center with its axis coinciding with that of the cell. The cell radius was chosen to be large enough not to affect the polymer conformation. The inner cylinder has radius $R=10 \AA$ and a linear charge density on its axis given by $\lambda=n e / l_{B}$, where $e$ is the elementary charge, and $l_{B}$ is the Bjerrum length. The parameter $n$ ranges from 2 to 6 .

The polyampholyte chain was represented by a neutral chain of 104 hard spheres (monomers) with radius $a=2 \AA$ and central charge $Q_{ \pm}= \pm e$. The monomers are connected by a harmonic potential $U_{h}(r)=0.5 k r^{2}$, where $r$ is the distance between the centers of two consecutive monomers, and $k=0.032 \mathrm{~N} / \mathrm{m}$. This set of parameters results in an average monomer-monomer distance of about $7.8 \AA$. Three types of chains composed of positive and negative blocks distributed sequentially were considered: diblock with 52 monomers each block, tetrablock with 26 monomers each block and octoblock with 13 monomers each block. Some results for alternating polyampholytes will be also presented [59].

The electrolyte solution was treated according to the Debye-Hückel theory, where the interaction between all pairs of charged monomers is simply given by the screening Coulomb potential with a hard-core restriction:

$$
U_{m m}(r)= \begin{cases}\infty, & r<2 a, \\ \frac{Q_{i} Q_{j} \exp (-\kappa r)}{4 \pi \epsilon_{0} \epsilon_{s} r}, & \text { otherwise }\end{cases}
$$

where $r$ is the distance between the centers of $i$ th and $j$ th monomers. The solvent dielectric constant is $\epsilon_{s}=78.7$, taken as that of the water at temperature $T=298.15 \mathrm{~K}$, $\epsilon_{0}$ is the vacuum dielectric permittivity and $\kappa^{-1}$ is the Debye screening length defined as a function of bulk salt concentration:

$$
\kappa=\left[\frac{e^{2}}{\epsilon_{0} \epsilon_{s} k_{B} T} \sum_{i} z_{i}^{2} n_{i 0}\right]^{\frac{1}{2}}
$$

where $k_{B}$ is the Boltzmann constant, $z_{i}$ is the valency of the ion $i$, and $n_{i 0}$ is the bulk number density of the $i$-th ionic species. The interaction monomer-cylinder is given by the solution of the cylindrical linear Poisson-Boltzmann equation:

$$
U_{m c}(r)= \begin{cases}\infty, & r<R+a, \\ \frac{Q_{i} \lambda}{2 \pi \epsilon_{0} \epsilon_{s} \kappa R_{m}} \frac{K_{0}(\kappa r)}{K_{1}\left(\kappa R_{m}\right)}, & \text { otherwise }\end{cases}
$$

where $r$ is the distance between the center of $i$-th monomer and the cylinder axis. $K_{0}(x)$ and $K_{1}(x)$ are the modified Bessel functions of the second kind [60].

In order to sample the polymer configurations, we have performed Metropolis Monte Carlo simulations, in NVT ensemble, using three movements: 1) the translational displacement of a single monomer randomly chosen, 2) the translational displacement of the whole chain and 3) the pivot rotation [61]. In each Monte Carlo step, one of these 

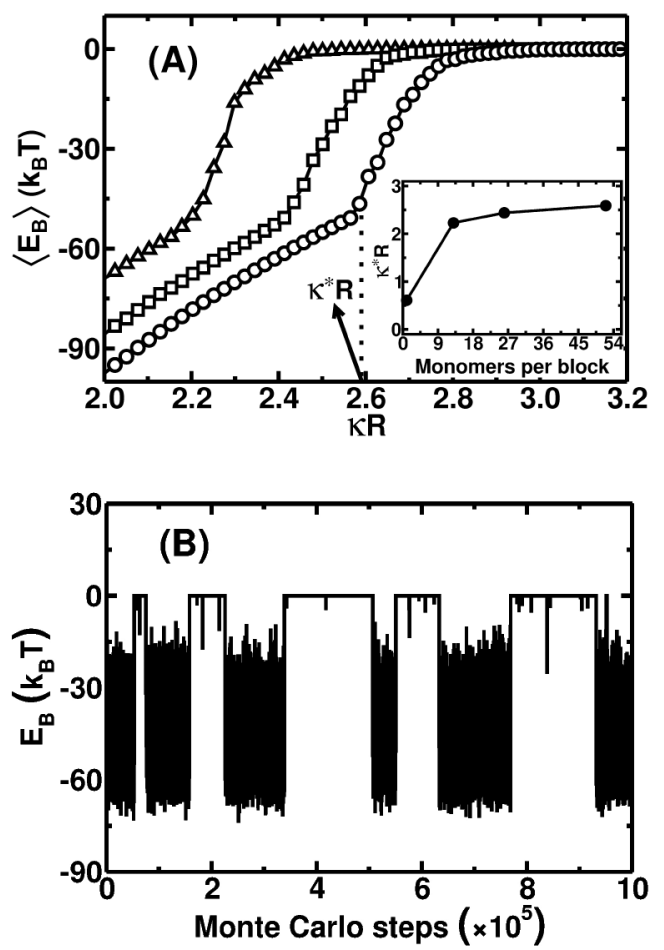

Fig. 1. (A) Mean binding energy, $\left\langle E_{B}\right\rangle$, as a function of $\kappa R$ for diblock (circles), tetrablock (squares) and octoblock (triangles). (B) Trajectory of the binding energy, $E_{B}$, for diblock structure in $\kappa R=2.67$ (that is equivalent to a salt concentration equal to $660 \mathrm{mM}$ ). In both plots, the cylinder linear charge density is $n=6$. The inset in plot (A) shows the dependence of $\kappa^{*} R$ on the number of monomers per block.

movements is randomly chosen, with probability equal to 0.9 for the first movement and 0.05 for the other ones. The equilibration process was carried out with $10^{6}$ Monte Carlo steps, and the average properties were calculated using $5 \times 10^{6}$ statistically uncorrelated configurations.

\section{Results and discussion}

In order to study the conformational properties of the adsorbed polyampholyte chain, it is necessary to check in which ionic strength conditions the adsorption is stable. Figure 1(A) shows the mean binding energy, $\left\langle E_{B}\right\rangle$, as a function of $\kappa R$ for the cylinder linear charge density $n=6$. This high charge density is close to that of mica and polystyrene latex particles $[62,63]$. The behavior we found is similar to that observed for charged homopolymers $[58,64,65]$. In low ionic strength, the chain is adsorbed onto the cylinder and $\left\langle E_{B}\right\rangle$ grows with increasing $\kappa$ due to the electrostatic screening. By adding salt, it is possible to identify a $\kappa^{*}$ value such that, for $\kappa>\kappa^{*}$, there is a more pronounced increasing of $\left\langle E_{B}\right\rangle$. This change in the trend growth of $\left\langle E_{B}\right\rangle$ is caused by the onset of coexistence of adsorbed (binding energy $E_{B}<0$ ) and desorbed $\left(E_{B} \approx 0\right)$ states, as can be seen in fig. 1(B). We also note that polyampholytes with distinct charge distribution remain adsorbed at different ranges of ionic

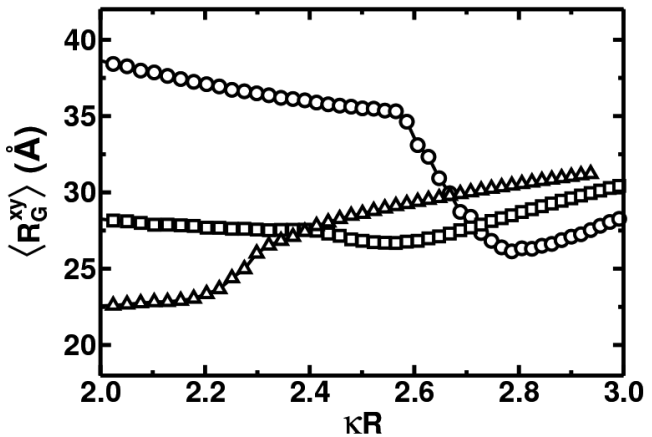

Fig. 2. Mean radius of gyration normal to the surface, $\left\langle R_{G}^{x y}\right\rangle$, as a function of $\kappa R$ for diblock (circles), tetrablock (squares) and octoblock (triangles). The cylinder linear charge density is $n=6$.

strength. The mean binding energy decreases for polymers with larger blocks, and then the $\kappa^{*}$ increases. This can be seen from the inset of fig. 1(A), which also depicts the results for the extreme case of alternating polyampholytes. In this regime of small block length, the ionic strength that leads to desorption decreases substantially with decreasing block length. The adsorption is stable only for high cylinder charge density and low salt concentration. Therefore, we investigate the effects of salt concentration and cylinder linear charge density on conformational properties of the polyampholyte under conditions in which $\kappa<\kappa^{*}$ for diblock, tetrablock and octoblock structures.

The transitions between the adsorbed and desorbed states also affect the component of radius of gyration normal to the surface, $\left\langle R_{G}^{x y}\right\rangle$. This effect can be observed in fig. 2 for the same parameters used in fig. 1(A). At low ionic strength, this component of the radius of gyration increases for polymers with larger blocks. In the case of diblock, the negative monomers form a large tail toward the solution (see also fig. 3(E)) due to the monomer-cylinder repulsion and the electrostatic rigidity of the chain, which contributes predominantly to the high value of $\left\langle R_{G}^{x y}\right\rangle$. Tetrablock and octoblock exhibit a successive decreasing of $\left\langle R_{G}^{x y}\right\rangle$ at this regime of low salinity due to the smaller size of the negative blocks, which form smaller loops and tails. Therefore, for $\kappa<\kappa^{*}$, the normal radius of gyration is predominantly determined by negative monomers and their distribution along the chain. When the salt concentration reaches a value in which $\kappa=\kappa^{*}$, the transitions between the adsorbed and desorbed states lead to an abrupt decreasing of the radius of gyration for the diblock, a slightly decreasing for the tetrablock, and an increasing for the octoblock. It is also observed an inversion of the dependence of the normal radius of gyration on the block size. For salinity conditions where the polymer is free in solution, the normal radius of gyration decreases for polymers with larger blocks, in agreement with results from simulations presented in ref. [52].

The distribution of monomers around the cylinder can be evaluated from fig. 3 . The average number of positive monomers, $n_{+}(r)$, as a function of $r /(R+a)$, where $r$ is the radial distance from the cylinder axis, is shown in figs. 3(A)-(D) for the cylinder charge density $n=6$ 

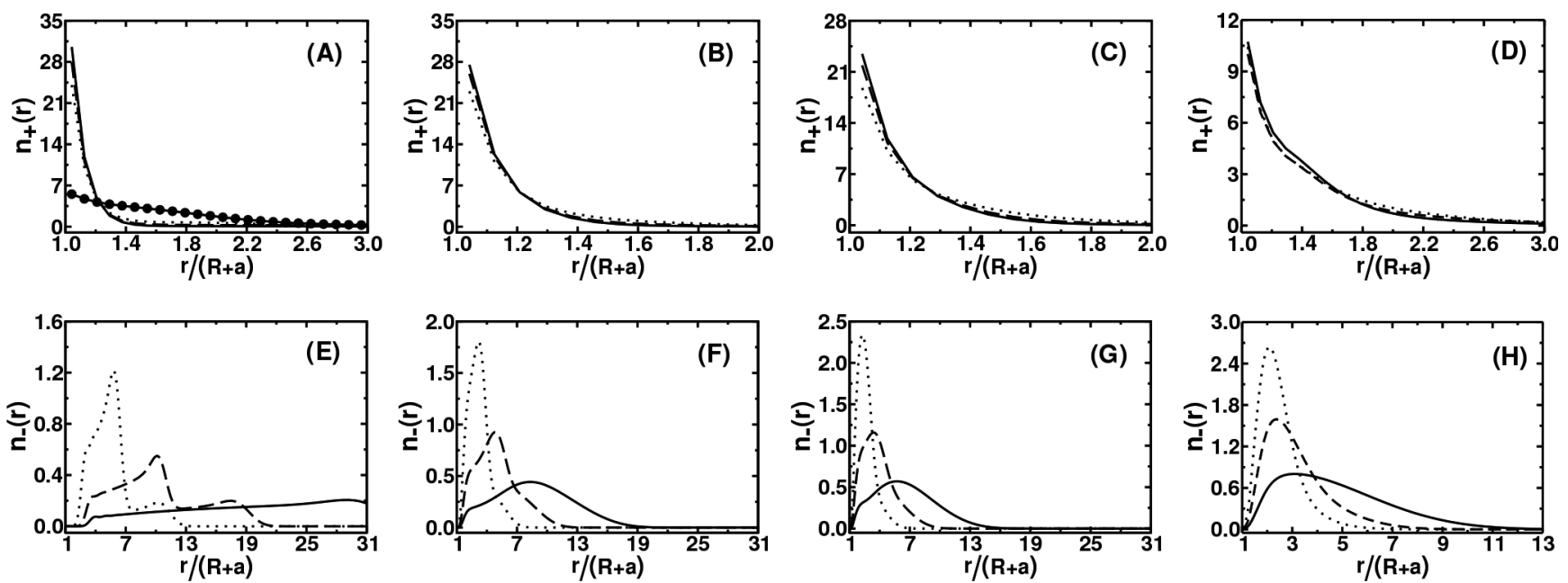

Fig. 3. Average number of positive, $n_{+}(r)$, and negative, $n_{-}(r)$, monomers as a function of $r /(R+a)$ for salt concentrations of $1 \mathrm{mM}$ (plots (A) and (E)), $50 \mathrm{mM}$ (plots (B) and (F)), $150 \mathrm{mM}\left(\right.$ plots $(\mathrm{C})$ and $(\mathrm{G})$ ), and for $\kappa=\kappa^{*}(\mathrm{plots}(\mathrm{D})$ and $(\mathrm{H}))$. The cylinder linear charge density is $n=6$ and the block structures are diblock (solid line), tetrablock (broken line) and octoblock (dotted line). The black spheres in plot (A) show the average number of positive monomers for the alternating polyampholyte.
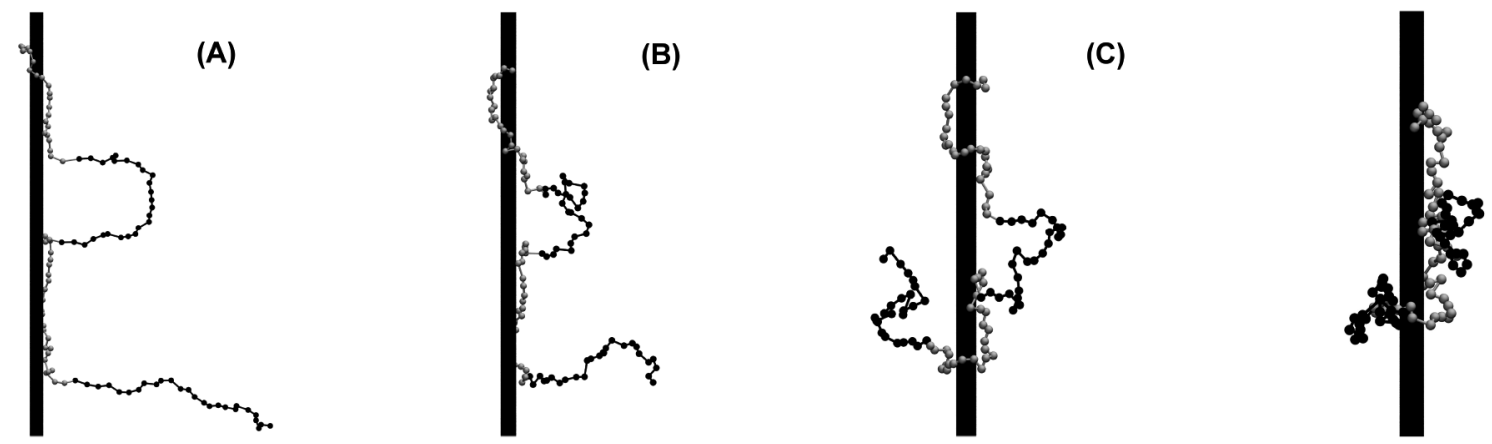

(D)

Fig. 4. Snapshots of equilibrium configurations of the tetrablock for four different salt concentrations: (A) $1 \mathrm{mM}$; (B) $50 \mathrm{mM}$, (C) $150 \mathrm{mM}$ and; (D) $\kappa=\kappa^{*}(540 \mathrm{mM})$. The cylinder linear charge density is $n=6$. The negative and positive monomers are represented by dark and light spheres, respectively.

and three different salt concentrations $(1,50$ and $150 \mathrm{mM})$ as well as at the desorption threshold $\left(\kappa=\kappa^{*}\right)$. In general, the increase in ionic strength causes the decrease of $n_{+}(r)$ in contact with the surface due to the screening of the electrostatic attractive interaction between the positive monomers and the cylinder. This interaction attenuation makes it no longer possible to overcome the entropic penalty to keep monomers in contact with the cylinder, resulting in the increase of positive loops, which contribute to the slight enlargement in the profile of distribution of positive monomers. These effects can also be observed in the snapshots in figs. 4(A)-(D) for the tetrablock. Figure $3(\mathrm{~A})$ also depicts results for the alternating polyampholyte, for a salt concentration equal to $1 \mathrm{mM}$. In this case, the negative monomer distribution profile is similar to the positive one. We can see that the number of monomers in contact with the cylinder decreases about 4 times, which results in the increase of the thickness of the distribution profile.

The average number of negative monomers, $n_{-}(r)$, located in the neighborhood of the cylinder is shown in figs. $3(\mathrm{E})-(\mathrm{H})$. The increase in the electrostatic screening allows negative monomers to come closer to the cylindrical surface. This behavior is due to the reduction of the electrostatic repulsion between the cylinder and the negative monomers as well as to the monomer-monomer interaction inside the same block, which is responsible for forming stretched tails or large loops at low ionic strength. At low salt concentration $(1 \mathrm{mM})$, the tetrablock and the octoblock present two regions with one peak on the monomer distribution profile. The region located at closer distances from the cylinder surface refers to large loops, whereas the other one is related to negative monomers in tails (see the snapshot in fig. 4(A)). Increasing the ionic strength, loops and tails assume similar lengths due to the decrease of the electrostatic persistence length and these two regions are no longer observed. At salt concentrations close to the adsorption threshold, negative and positive charged monomers, which are close to the block junction, interact more strongly with each other than with the cylinder. Due to this effect, the polymer adopts a necklace-like conformation (see the snapshot in fig. 4(D)).

The effects of the cylinder charge density, $n$, can be evaluated in fig. 5 , where the average number of monomers 

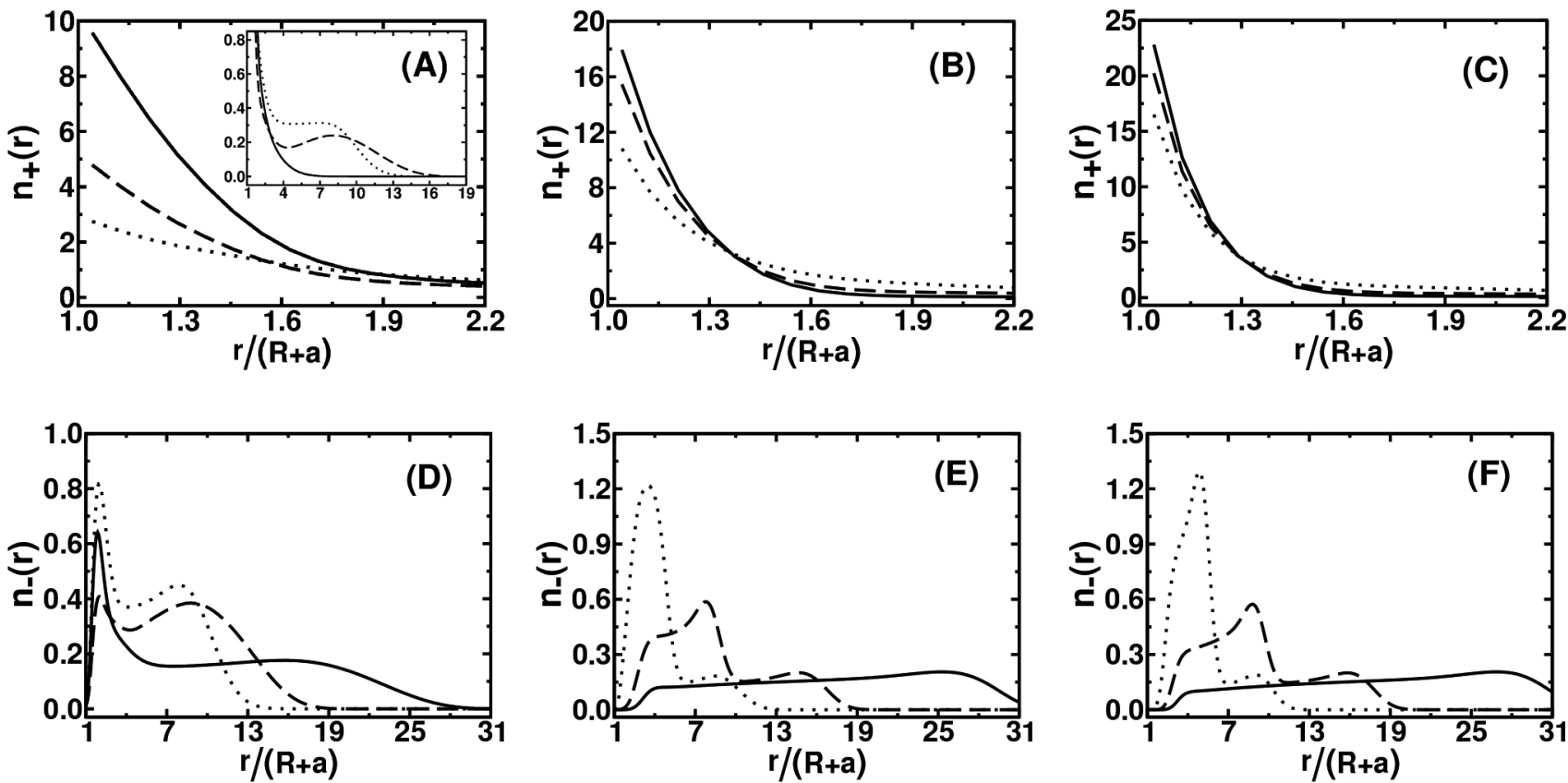

Fig. 5. Average number of positive, $n_{+}(r)$, and negative, $n_{-}(r)$, monomers, as a function of $r /(R+a)$ for salt concentration of $1 \mathrm{mM}$ and three different charge densities of the cylinder: (A) and (D) $n=2$; (B) and (E) $n=3$; and $(\mathrm{C})$ and $(\mathrm{F}) n=4$. The block structures are diblock (solid line), tetrablock (broken line) and octoblock (dotted line).

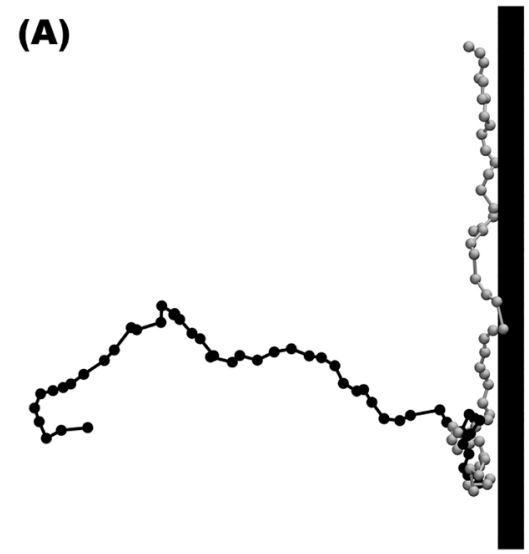

(B)

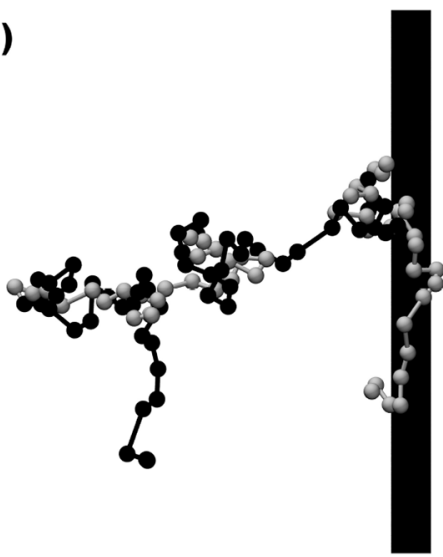

(C)

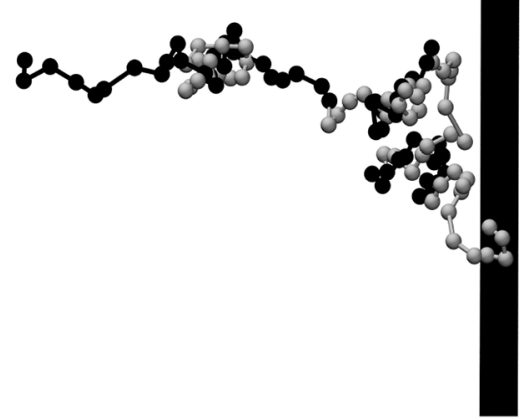

Fig. 6. Snapshots of equilibrium configurations in salt concentration of $1 \mathrm{mM}$ and cylinder linear charge density $n=2$ for three block structures of the polyampholyte: (A) diblock; (B) tetrablock; and (C) octoblock. In all figures, the negative and the positive monomers are represented by dark and light spheres, respectively.

as a function of $r /(R+a)$, for $n=2,3$ and 4 at low ionic strength $(1 \mathrm{mM})$, is shown. As already observed for reducing salt concentration, the increase of $n$ promotes the growth of the average number of positive monomers in contact with the cylinder surface (monomers in trains) and a slight decrease in the thickness of $n_{+}(r)$ (see figs. $5(\mathrm{~A})-(\mathrm{C}))$. The reduction of the charge density leads to the increasing variation of $n_{+}(r)$ as a function of the number of blocks of the polyampholyte. For $n=2$, the average number of positive monomers in trains increases in proportion to the size of the blocks. This can be understood from the snapshots in fig. 6, where it is shown that only a portion of the first positive block is kept in contact with the surface. Therefore, polyampholytes with larger blocks have more monomers in trains. Unlike what happens for $n>2$, a fraction of positive monomers is located beyond the first layer, close to the cylinder (see the inset in fig. 5(A)). As will be discussed below, this occurs because the chain assumes a necklace-like conformation normal to the surface.

Regarding the negative monomers, figs. 5(E) and (F) present, respectively, the results to $n=3$ and $n=4$ and they exhibit practically the same distribution profiles observed in fig. 3(E). For tetrablock and octoblock polyampholytes, the first peak is related to the negative monomers in loops, whereas the second one corresponds to the monomers in tail. The increase of the charge density causes a shift of the peaks to larger radial distances due to 

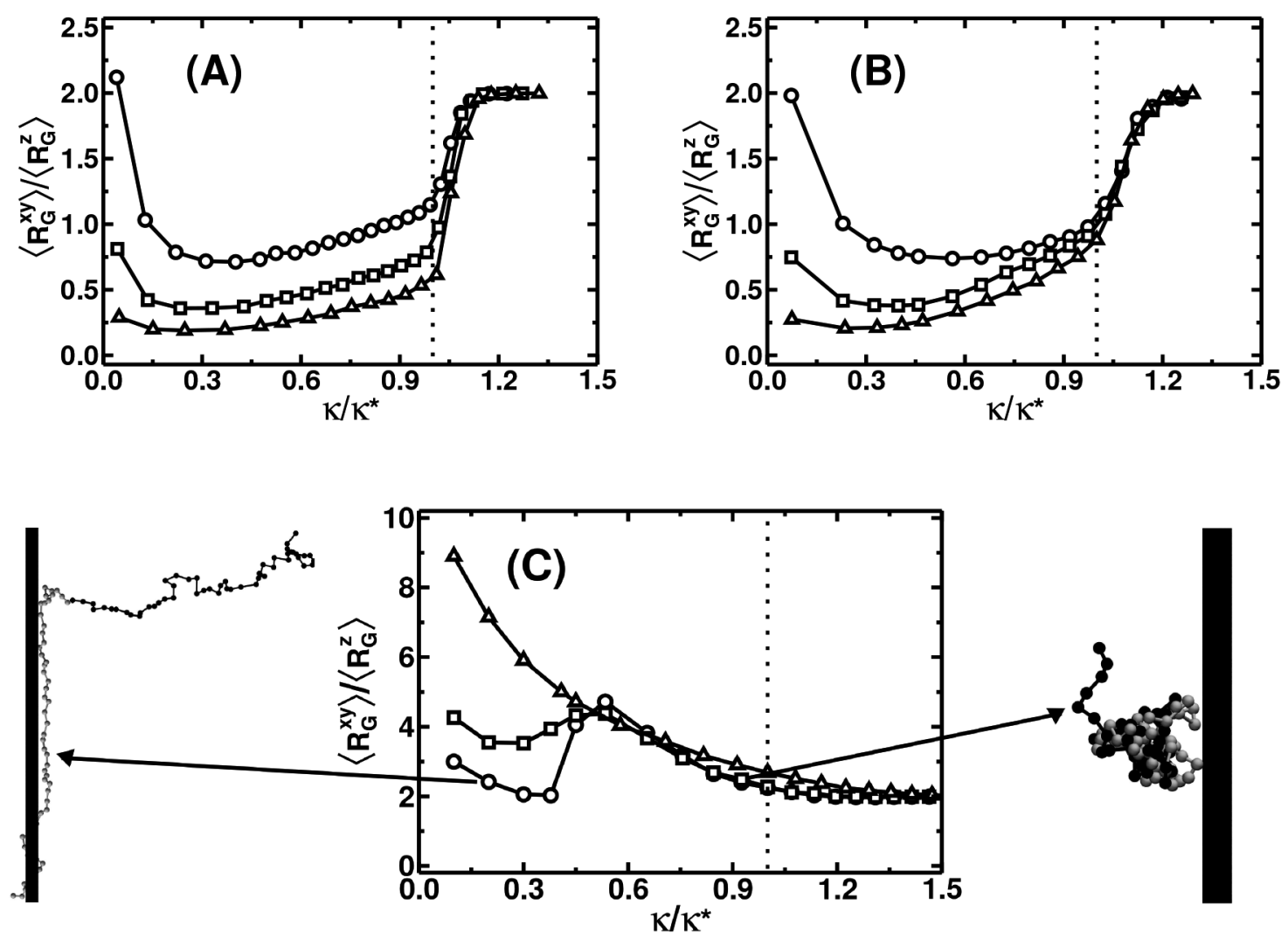

Fig. 7. Ratio between the normal and the lateral component of the radius of gyration, $\left\langle R_{G}^{x y}\right\rangle /\left\langle R_{G}^{z}\right\rangle$, as a function of $\kappa / \kappa^{*}$ for three distinct charge densities of the cylinder: (A) $n=6$; (B) $n=4$; and (C) $n=2$. The block structures are diblock (circles), tetrablock (squares) and octoblock (triangles). In all graphs, the vertical dotted line marks the desorption threshold $\left(\kappa=\kappa^{*}\right)$. The snapshots correspond to the diblock in salt concentrations indicated by the arrows, where dark and light spheres represent, respectively, the negative and the positive monomers.

the stronger electrostatic repulsion between the cylinder and the negative monomers. For $n=3$, the first peak is located at $r \approx 41 \AA$ and $93 \AA$ for the octoblock and the tetrablock, respectively, whereas for $n=4$, we have $r \approx 58 \AA$ and $105 \AA$. Considering the diblock, we can observe that the negative monomers form a stretched tail toward the solution. For $n=2$, the first peak is located very close to the surface for all block structures due to the attractive interaction between positive and negative monomers close to the block junction (see snapshots in fig. 6).

The orientation of the chain regarding the cylinder axis can be evaluated by the ratio between the normal, $\left\langle R_{G}^{x y}\right\rangle$, and the lateral, $\left\langle R_{G}^{z}\right\rangle$, components of the radius of gyration. Figures $7(\mathrm{~A})-(\mathrm{C})$ show $r=\left\langle R_{G}^{x y}\right\rangle /\left\langle R_{G}^{z}\right\rangle$ as a function of $\kappa / \kappa^{*}$ for cylinder charge densities $n=2, n=4$ and $n=6$. At high salt concentrations $\left(\kappa>\kappa^{*}\right)$, the polyampholyte chain is desorbed and, then, $r=2$ showing that no direction is preferred. For $n=4$ and $n=6$, the chain is adsorbed predominantly parallel to the surface and then $r<2$, except for the diblock at low ionic strength. In this case, $r \approx 2$ due to the large tail which induces the chain to adopt an L-shape. Increasing $\kappa$ initially leads to a decrease of $r$ because the negative blocks become closer to the surface. It is then followed by a slight increase of $r$ caused by the decrease in the lateral size of the chain. Even close to the desorption threshold (see the vertical dotted line, at $\kappa=\kappa^{*}$, in fig. 7), the polymer assumes a conformation predominantly parallel to the surface. Decreasing the cylinder charge density to $n=2$, the behavior of $r$ is quite distinct and indicates that the polymer is adsorbed predominantly normal to the surface. For the diblock, the increase in salt concentration promotes an abrupt variation of $r$ at $\kappa / \kappa^{*} \approx 0.4$. This variation is associated with a conformational transition from an L-shape to a conformation in which the two blocks interact strongly. A smoother transition is also observed for the tetrablock at the same ionic strength regime. In these conditions, the conformation at low ionic strength is like a necklace normal to the surface. Increasing the salt concentration leads to a globular conformation. Unlike what happens for $n=4$ and 6 , when $\kappa / \kappa^{*}$ approaches to unity, no abrupt variations of $r$ are observed, indicating that the adsorbed polyampholyte at the desorption threshold assumes a globular conformation similar to that one when free in solution. 


\section{Concluding remarks}

The electrostatic interaction between a negatively charged cylinder and block-polyampholytes was studied by means of Metropolis Monte Carlo simulations. The range of the charge density of the chosen cylinder ( $n$ ranging from 2 to 6) enabled us to study the effects on the adsorbed chain, with different block sizes, in a properly large range of salt concentration. For the lower charge density considered $(n=2)$, the polyampholyte assumes, at low salt concentration, a necklace-like conformation normal to the surface. In this case, the length of the adsorbed chain increases with the increase of the block size. Increasing $n$ in this same low ionic strength regime, the conformation of the chain changes and it is now characterized by a large tail and large loops negatively charged and by the positive monomers within a region of size smaller than $R / n$, as in the pancake regime. In this last case, the thickness of the adsorbed layer is determined by the size of the loops and so it increases with the size of the blocks. Increasing the salt concentration, the thickness decreases because of the shrinkage of the loops and, then, the pancake-like shape changes to a necklace-like conformation parallel to the surface of the cylinder due to the interaction among the oppositely charged monomers that are close to the block junction. In spite of this conformation being characteristic of charged polyampholytes in solution, it is assumed by neutral polyampholytes on the surface. This occurs in order to balance the monomer-monomer interaction inside the chain, without which there would be a globular conformation and, consequently, a decrease of the contact of positive monomers with the cylinder.

According to the theoretical approach described by Netz and Joanny [45] for random polyampholytes, the adsorption on cylindrical surfaces can be mapped from the planar case, replacing the Gouy-Chapmann length, $\lambda$, by $R / n$, where $R$ is the cylinder radius. The adsorption diagram for the planar case presented in fig. 6 of ref. [43] predicts that the increase in salt concentration leads to a weak adsorption regime, independently of the cylinder charge density. This behavior has been observed only for low cylinder charge density $(n=2)$. For higher values of $n$, the polyampholyte undergoes a conformational transition from an adsorbed necklace-like conformation to a globular one.

This research was supported by computational resources supplied both by João Ruggiero Neto and the Center of Scientific Computing (NCC/GridUNESP) of the Sao Paulo State University (UNESP). DLZC thanks the Sao Paulo Research Foundation (FAPESP, Process Grant No. 2013/13151-7 and 2015/03549-9) and the Coordination for the Improvement of Higher Education Personnel (CAPES) for the financial support.

\section{Author contribution statement}

Daniel L.Z. Caetano performed all computer simulations and wrote the manuscript. Sidney J. de Carvalho reviewed the manuscript and supervised the research work.

\section{References}

1. J.F. Joanny, J. Phys. II 4, 1281 (1994).

2. R.R. Netz, D. Andelman, Phys. Rep. 380, 1 (2003).

3. R. de Vries, M.C. Stuart, Curr. Opin. Colloid Interface Sci. 11, 295 (2006).

4. S. Ulrich, M. Seijo, S. Stoll, Curr. Opin. Colloid Interface Sci. 11, 268 (2006).

5. A.V. Dobrynin, Curr. Opin. Colloid Interface Sci. 13, 376 (2008).

6. R.G. Winkler, A.G. Cherstvy, in Polyelectrolyte Complexes in the Dispersed and Solid State I (Springer, 2013) pp. 156 .

7. K.W. Mattison, P.L. Dubin, I.J. Brittain, J. Phys. Chem. B 102, 3830 (1998).

8. F. Ozon, J.M. di Meglio, J.F. Joanny, Eur. Phys. J. E 8, 321 (2002).

9. A. Laguecir, S. Stoll, G. Kirton, P. Dubin, J. Phys. Chem. B 107, 8056 (2003).

10. A. Cherstvy, R. Winkler, J. Chem. Phys. 125, 064904 (2006).

11. C.L. Cooper, A. Goulding, A.B. Kayitmazer, S. Ulrich, S. Stoll, S. Turksen, S.i. Yusa, A. Kumar, P.L. Dubin, Biomacromolecules 7, 1025 (2006).

12. G. Decher, Science 277, 1232 (1997).

13. F. Caruso, R.A. Caruso, H. Möhwald, Science 282, 1111 (1998).

14. B.G. De Geest, S. De Koker, G.B. Sukhorukov, O. Kreft, W.J. Parak, A.G. Skirtach, J. Demeester, S.C. De Smedt, W.E. Hennink, Soft Matter 5, 282 (2009).

15. V. Trubetskoy, D. Rozema, S. Monahan, V. Budker, J. Hagstrom, J. Wolff et al., Delivery of sirna to cells using polyampholytes, uS Patent App. 10/368,139 (2003).

16. V.S. Trubetskoy, J.E. Hagstrom, V.G. Budker, J.A. Wolff, D.B. Rozema, S.D. Monahan, ph-titratable polyampholytes for delivering polyions to a cell, uS Patent 7,033,607 (2006).

17. Z. Iatridi, G. Mattheolabakis, K. Avgoustakis, C. Tsitsilianis, Soft Matter 7, 11160 (2011).

18. Q. Yi, D. Wen, G.B. Sukhorukov, Langmuir 28, 10822 (2012).

19. M.A. Wolfert, E.H. Schacht, V. Toncheva, K. Ulbrich, O. Nazarova, L.W. Seymour, Hum. Gene Ther. 7, 2123 (1996).

20. V.S. Trubetskoy, A. Loomis, J.E. Hagstrom, V.G. Budker, J.A. Wolff, Nucleic Acids Res. 27, 3090 (1999).

21. A. Elouahabi, J.M. Ruysschaert, Mol. Ther. 11, 336 (2005).

22. L. Medina-Kauwe, J. Xie, S. Hamm-Alvarez, Gene Ther. 12, 1734 (2005).

23. A.V. Dobrynin, R.H. Colby, M. Rubinstein, J. Polym. Sci., Part B: Polym. Phys. 42, 3513 (2004).

24. J. Bohrisch, C.D. Eisenbach, W. Jaeger, H. Mori, A.H. Müller, M. Rehahn, C. Schaller, S. Traser, P. Wittmeyer, in Polyelectrolytes with Defined Molecular Architecture I (Springer, 2004) pp. 1-42.

25. D.H. Napper, Polymeric Stabilization of Colloidal Dispersions, Vol. 3 (Academic Press, 1983).

26. J. Buffle, K.J. Wilkinson, S. Stoll, M. Filella, J. Zhang, Environ. Sci. Technol. 32, 2887 (1998).

27. S.Z. Zu, B.H. Han, J. Phys. Chem. C 113, 13651 (2009).

28. S. Asayama, M. Nogawa, Y. Takei, T. Akaike, A. Maruyama, Bioconjug. Chem. 9, 476 (1998). 
29. F. Von Goeler, M. Muthukumar, J. Chem. Phys. 100, 7796 (1994).

30. L. Gutman, A.K. Chakraborty, J. Chem. Phys. 101, 10074 (1994).

31. R.R. Netz, J.F. Joanny, Macromolecules 32, 9013 (1999).

32. K.K. Kunze, R.R. Netz, Phys. Rev. Lett. 85, 4389 (2000).

33. A. Shafir, D. Andelman, R.R. Netz, J. Chem. Phys. 119, 2355 (2003).

34. A. Shafir, D. Andelman, Phys. Rev. E 70, 061804 (2004).

35. A.G. Cherstvy, R.G. Winkler, J. Chem. Phys. 120, 9394 (2004).

36. R.G. Winkler, A.G. Cherstvy, Phys. Rev. Lett. 96, 066103 (2006).

37. R.G. Winkler, A.G. Cherstvy, J. Phys. Chem. B 111, 8486 (2007).

38. A. Cherstvy, R. Winkler, Phys. Chem. Chem. Phys. 13, 11686 (2011).

39. H. Boroudjerdi, A. Naji, R.R. Netz, Eur. Phys. J. E 37, 21 (2014).

40. S.J. de Carvalho, R. Metzler, A.G. Cherstvy, Phys. Chem. Chem. Phys. 16, 15539 (2014)

41. S.J. de Carvalho, R. Metzler, A.G. Cherstvy, Soft Matter 11, 4430 (2015)

42. S.J. de Carvalho, R. Metzler, A.G. Cherstvy, New J. Phys. 18, 083037 (2016).

43. A.V. Dobrynin, M. Rubinstein, J.F. Joanny, Macromolecules 30, 4332 (1997).

44. S. Neyret, L. Ouali, F. Candau, E. Pefferkorn, J. Colloid Interface Sci. 176, 86 (1995).

45. R.R. Netz, J.F. Joanny, Macromolecules 31, 5123 (1998).

46. G.S. Manning, J. Chem. Phys. 51, 924 (1969).

47. E. Zhulina, A. Dobrynin, M. Rubinstein, Eur. Phys. J. E 5, 41 (2001).
48. A.V. Dobrynin, E.B. Zhulina, M. Rubinstein, Macromolecules 34, 627 (2001).

49. A.V. Dobrynin, Phys. Rev. E 63, 051802 (2001).

50. E.B. Zhulina, A.V. Dobrynin, M. Rubinstein, J. Phys. Chem. B 105, 8917 (2001)

51. M.O. Khan, T. Åkesson, B. Jönsson, Macromolecules 34, 4216 (2001)

52. R. Messina, Eur. Phys. J. E 22, 325 (2007).

53. J. Feng, E. Ruckenstein, Polymer 44, 3141 (2003).

54. S. Ulrich, M. Seijo, F. Carnal, S. Stoll, Macromolecules 44, 1661 (2011)

55. F. Carnal, A. Clavier, S. Stoll, Environ. Sci.: Nano 2, 327 (2015).

56. H. Chen, E. Ruckenstein, Langmuir 30, 3723 (2014).

57. H. Wennerström, B. Jönsson, P. Linse, J. Chem. Phys. 76, 4665 (1982)

58. S.J. De Carvalho, D.L.Z. Caetano, J. Chem. Phys. 138, 244909 (2013).

59. J. Wittmer, A. Johner, J. Joanny, Europhys. Lett. 24, 263 (1993).

60. M. Abramowitz, I.A. Stegun, Handbook of Mathematical Functions: with Formulas, Graphs, and Mathematical Tables, Vol. 55 (Courier Corporation, 1964).

61. K. Binder, Monte Carlo and Molecular Dynamics Simulations in Polymer Science (Oxford University Press, 1995).

62. W.M. Brouwer, R.L. Zsom, Colloids Surf. 24, 195 (1987).

63. O.J. Rojas, Adsorption of polyelectrolytes on mica, in Encyclopedia of Surface and Colloid Science, Vol. 1 (Marcel Dekker, Inc., 2002) p. 517.

64. S. de Carvalho, EPL 92, 18001 (2010).

65. V. de Oliveira, S. de Carvalho, Eur. Phys. J. E 37, 75 (2014). 